\title{
Realization of prediction of materials properties by ab initio computer simulation
}

\author{
YOSHIYUKI KAWAZOE \\ Institute for Materials Research (IMR), Tohoku University, Sendai 980-8577, Japan
}

\begin{abstract}
A b$ initio treatment is becoming realistic to predict physical, chemical, and even mechanical properties of academically and industrially interesting materials. There is, however, some limitation in size and time of the system up to the order of several hundred atoms and $\sim 1$ pico second, even if we use the fastest supercomputer efficiently. Therefore, it is very difficult to simulate realistic materials with grain boundaries and important reactions like diffusion in materials. To improve this situation, two ways have been invented. One way is to upgrade approximations to match the necessary levels according to inhomogeneous electron gas theory beyond the present day standard, i.e. local density approximation (LDA). The reason is simply that the system we are interested in is composed of many particles interacting with Coulomb forces governed by quantum mechanics. (Complete knowledge is available, and only what we should do is to make better approximations to explain the phenomena!). Another is to extract the necessary parameters from the ab initio calculations on systems with limited number of atoms, and apply these results into cluster variation, direct, or any other sophisticated methods based on classical concepts such as statistical mechanics.

In this paper, several typical examples recently worked out by our research group are introduced to indicate that these methodologies are actually possible to be successfully used to predict materials properties before experiments based on the present day state-of-art supercomputing systems. It includes scientific visualization of the results of $a b$ initio molecular dynamics simulation on atom insertion process to $C_{60}$ and to carbon nanotube, tight-binding calculation of single electron conductance properties in nanotube to create nano-scale diode virtually by computer, which will be a base of future nanoscale electric device in nanometer size, $\mathrm{Li}+\mathrm{H}$ reaction without Born-Oppenheimer approximation, structural phase transitions in perovskite materials under very high pressure in earth by direct method, and prediction of wavelength of emitted light from Na clusters with GW ( $G=$ Green function-vertex, $W=$ screened Coulomb interaction) approximation.
\end{abstract}

Keywords. Materials properties; ab initio computer simulation.

\section{Introduction}

It has been studied mainly experimentally to realize useful materials properties, because they are very complex in nature and simple models or quantum mechanics have not been able to predict them. On the contrary, theoretical treatments based on the band structure calculations with local density approximation (LDA) have been applied mainly to solve much simpler problems, where the electronic structures are calculated assuming the crystal structures, which are determined traditionally by the $\mathrm{X}$ ray experiments for sample single crystals. It has been too time consuming to determine the crystal structures by $a b$ initio method without the experimental information beforehand even if we used the supercomputer. This situation, however, has been dramatically changed recently because the processing speed and the memory size of the supercomputer have reached to the range of 1TFLOPS (Tera FLoating Operation Per Second) and 1TB (Tera Byte), respectively, so that we can now apply $a b$ initio methods to realistic materials. Also there have been new inventions of theoretical tools and algorithms such as the
Car-Parrinello (CP) method, that reduce the necessary computer resources dramatically (Ohno et al 1999). In the present paper, recent results based on the mixed-basis all-electron formulation are introduced focusing on two interesting examples: (i) $\mathrm{GW}$ approximation applied to micro-clusters to estimate absolute highest occupied molecular orbital (HOMO) - lowest unoccupied molecular orbital (LUMO) gap to predict the expected wavelength of the light emitted from cluster assembled new materials, and (ii) time-dependent Schrödinger equation solved to simulate chemical reactions passing through out of the Born-Oppenheimer surface.

Figure 1 shows a schematic diagram on the classification of $a b$ initio calculation methods. Upper part in the figure includes the present day standards like LDA, LSDA (local spin density approximation), GGA (generalized gradient approximation), $\mathrm{CP}$, and MO (molecular orbital calculation). These methods are basically based on the single electron approximation and exchange and correlation effects are treated in this scheme. The lower part is for the research and developmental levels like GW (Green function vertex correction), TD-Sch (time dependent Schrödinger equation solver), which will be intro- 
duced in this talk. Quantum Monte Carlo ( $q$-MC) is a variational approach and it includes full exchange-correlations, but it is difficult to certify that the ground state is achieved.

\section{Underlying physical and chemical principles- mixed-basis all-electron full-potential formulation}

It is very often necessary to employ all-electron fullpotential formalism to simulate materials properties in detail. For example, the hyperfine structure can only be estimated with the core contribution, XPS experiments also measure the deep levels, and effective pseudopotential is very difficult to be made for the 2 nd row elements like carbon. Up to the present, full-potential linearized augumented plane wave (FLAPW) method has been developed and used widely (Ohno et al 1999). This method, however, requires extremely large computational resources. Blow, a new computational scheme used in an all-electron full-potential mixed-basis approach, which aims to reduce necessary computational resources keeping high numerical accuracy, is described briefly. The mixed-basis approach was originally introduced in the $a b$ initio calculation of transition metal elements by Louie et al (1979) in order to treat efficiently the spatial locality and asymmetry of $3 d$ orbitals with limited number of basis functions. It is a technique to expand the wave functions in terms of both plane waves (PW) and atomic orbitals (AO). They applied this approach in pseudopotential formalism. The pseudo-potential scheme allows one to replace a strong electron-ion Coulomb potential with a soft potential to reduce computational complexities. These important features of the all-electron fullpotential mixed-basis formulation are summarized in figure 2 .

However, it has often been pointed out that the description of wave functions only with PWs is not easy, in particular, for $2 p$ orbitals in the second-row elements and $3 d$ orbitals in transition metal elements, even if an efficient pseudo-potential is used. Accordingly, we have developed an original all-electron full-potential mixedbasis program; both PWs and AOs are used to expand the true wave functions including the core states.

This all-electron full-potential mixed-basis approach for band structure calculations was first applied to analyse the experimental results of aligned $\mathrm{C}_{60}$ on $\mathrm{Cu}(111)$

- Present standard first principles method

- LDA+GGA with pseudopotential+plane wave expansion

- The best treatment from our group: original formulation

- LDA(LSDA)+GGA

- all-electron full-potential by mixed-basis ( $\mathrm{PW}+\mathrm{AO}$ )

- equilibration for lattice and electrons (SD, CG)

- advantages: absolute energy, core-electron rehated properties, $2^{\text {ed }}$ row elements (C, O, N, organic materials)

- GW approximation

- Time dependent Schrödinger equation solver

Figure 2. Importance of all-electron formulation.

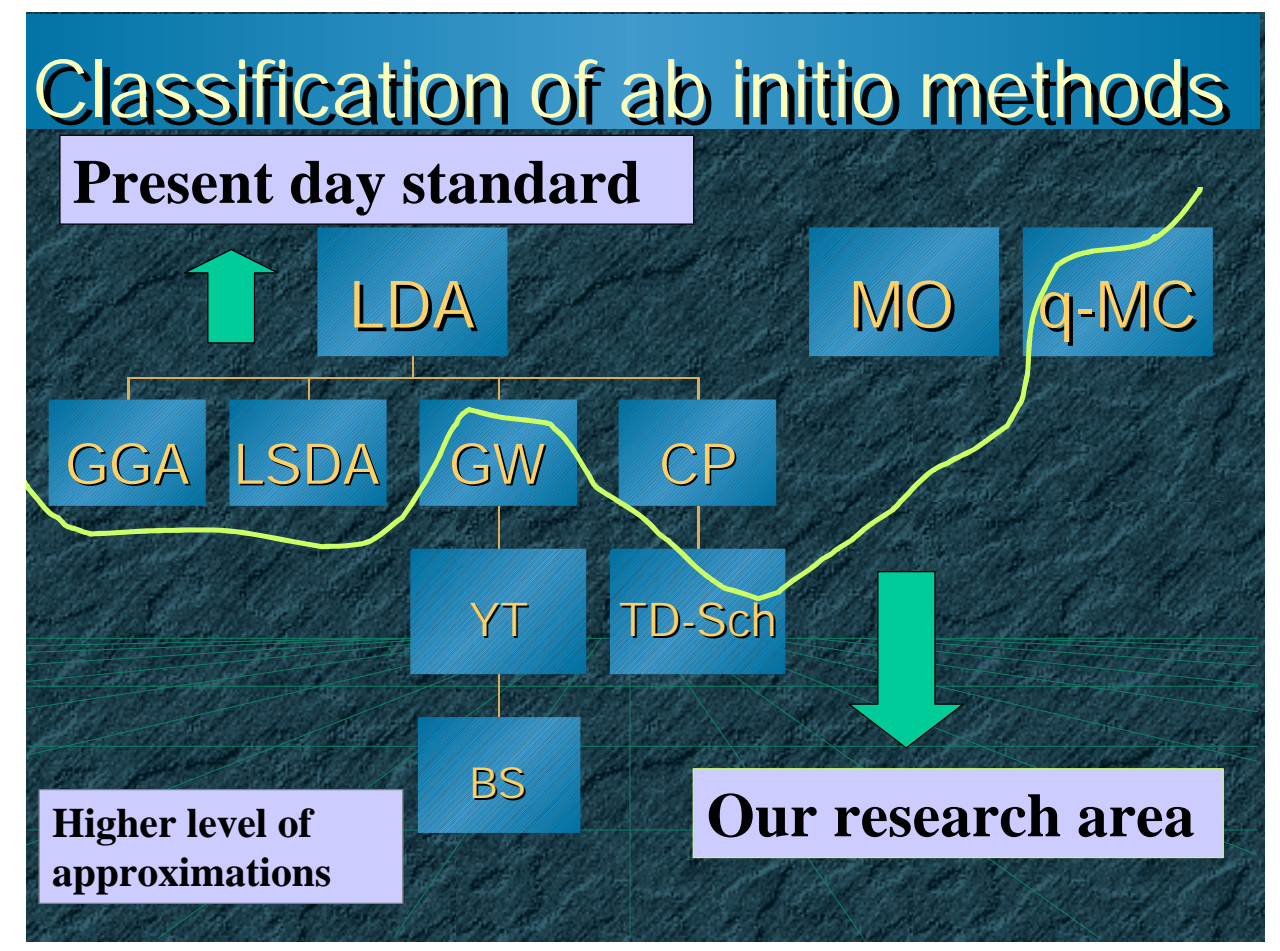

Figure 1. Classification of ab initio methods. 
successfully to reproduce the observed scanning tunneling microscopic (STM) images (Maruyama et al 1995). Car-Parrinello type molecular dynamics has also been performed to simulate the insertion processes of variety of atoms into several types of fullerenes (Ohno et al 1996). In the mixed-basis formalism, because AOs are not orthogonal to the PWs, it is necessary to orthogonalize the basis set at each iteration step in the simulation. The exchange-correlation potential is evaluated in real space. In the present formalism, the charge density and the self-consistent potential are evaluated in real space for AO-related parts and in Fourier space for PWrelated parts.

Once the potential is determined, the Hamiltonian matrix elements have to be evaluated. In the potential matrix elements, there are three types of combinations: PW-PW, PW-AO, and AO-AO. Similarly as in the original Car-Parrinello method with PW basis, it is not necessary to register the $\mathrm{PW}-\mathrm{PW}$ matrix elements in computer-memory, because the PW-PW block does not change after diagonal transformation. The other combinations $\mathrm{PW}-\mathrm{AO}$ and $\mathrm{AO}-\mathrm{AO}$ are conveniently calculated in real space inside each atomic sphere in each iteration.

This original all-electron full-potential mixed-basis code has been rewritten several times. The first version was developed with Slater orbitals. Secondly, the Herman-Skillman numerical wave functions were used to enhance the accuracy. Lastly, all the codes were converted from Fortran77 to Fortran90 to realize transferability. In the following sections, this all-electron fullpotential mixed-basis code was used as the fundamental tool in all the simulations (Sluiter et al, to be published).

\section{Current research results}

\subsection{Exact HOMO-LUMO gap value estimation for clusters by $G W$ approximation}

LDA is known to function very precisely to determine some of the ground state properties of crystals and clusters. It is, however, not possible to estimate excited state properties more than the Fermi level. The reason is simply that LDA is formulated based on the density functional theory (DFT), which assumes the variational theory to find out the minimum energy state, and accordingly the applicability is restricted to the ground state properties. Within DFT, one important progress is the generalized gradient approximation (GGA). It contributes significantly to the accurate determination of the structure of the ground state. For example, the ground state in $\mathrm{Fe}$ crystal is estimated to be an $f c c$ phase within LDA. It is completely wrong, and GGA helps to confirm it as a bcc phase correctly (Ohno et al 1999).

Most of the physical and chemical properties are related to the excited states of electrons. Electric and magnetic fields applied to materials make them excited in general. To analyse the observed properties theoretically, it is fundamentally necessary to treat excited states. For example, ionization potential is the energy to take one electron from the material. Electron affinity is the value to add one electron to the material. These values are determined by applying laser beam and study the ionization and/or neutralization. Therefore, they are only possible to be estimated by the scheme of quasi-particles; i.e. one electron added/subtracted from the medium. It is well known in the quantum many body theory that detailed behaviours of quasi-particles are determined by the time-dependent perturbation theory. The reason is that it takes a finite time for the light emission after the excitation, and it is a dynamic process for electron clouds.

As is well known LDA gives only around $50 \%$ of the observed band gap value for semiconductors. This big

\section{Quasi-particle approximation}

Louie group: crystal, pseudopotential

Present : any structure, all-electron

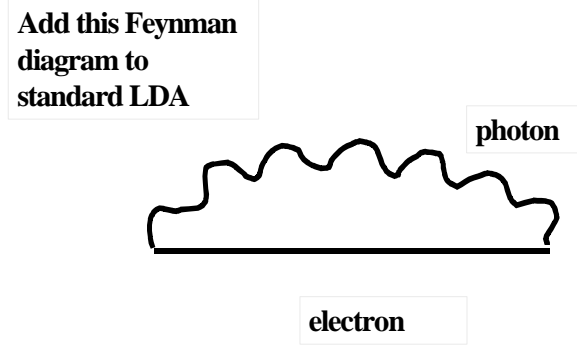

Figure 3. GW approximation which adds a quasiparticle Feynman diagram to standard LDA.

\section{GW approximation}

\section{Election self-energy in the first order perturbation}

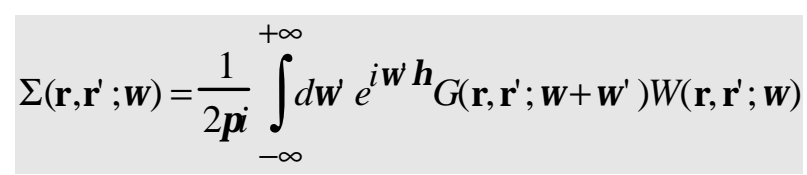
Here $G$ and $W$ are Green's function and dynamically screened Coulomb interaction, respectively. $\omega$ ' denotes frequency and $\eta$ a positive infinitestimal. $W$ is represented by dielectric response function $\varepsilon$ and bare Coulomb interaction v.

Figure 4. Self energy correction to LDA in GWA. 
discrepancy is caused by the fact that the one electron energy spectrum obtained by LDA does not express the quasi-particle spectrum. To overcome this problem, electron self-energy should be estimated precisely. Hedin (1965) first introduced the formulation called GW approximation (GWA), which is based on the 1st order perturbation theory. Here, G stands for the Green's function and $\mathrm{W}$ is for the screened Coulomb interaction. Within the random phase approximation (RPA), GW approximation is now possible to be applied to realistic materials. Figure 3 shows the Feynman diagram added in GWA to the standard LDA, and figure 4 the explicit expression of the self energy correction in GWA to LDA as a perturbation. Schematic presentation of the importance of GWA is shown in figure 5, which shows exact band gap estimation by GWA.

\section{Prediction of photoemission band gap value}

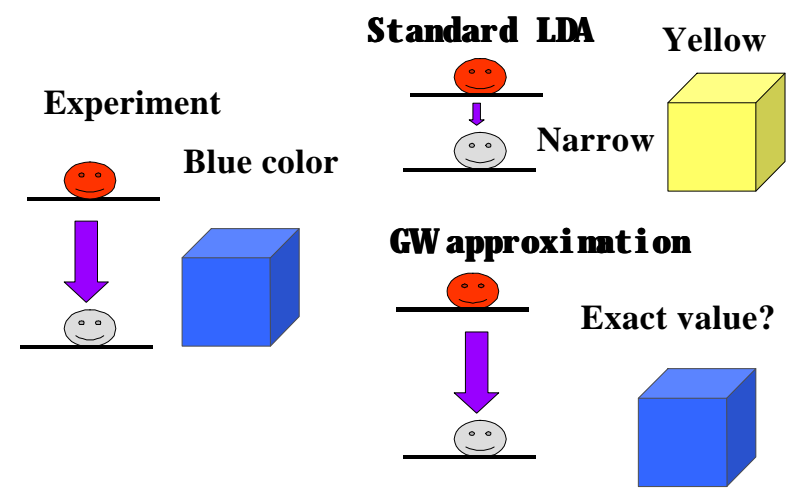

Figure 5. Prediction of exact band gap value by GW approximation.
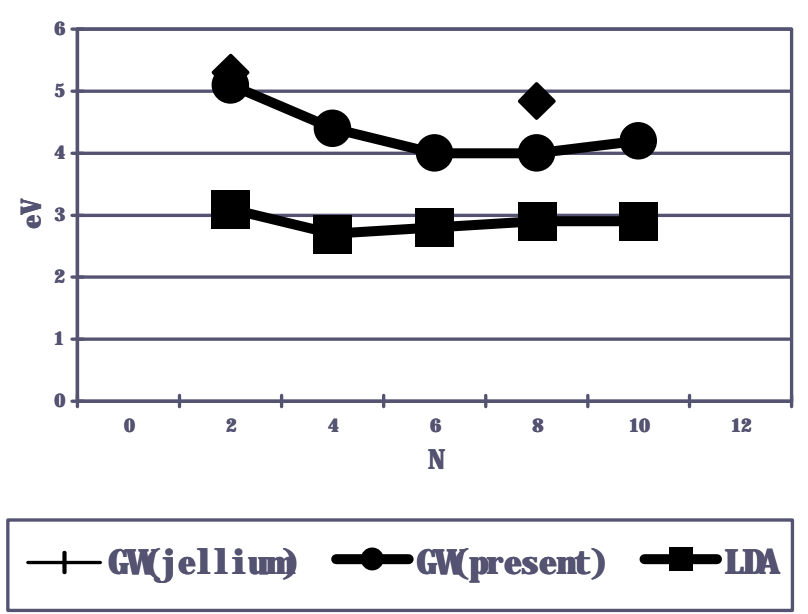

Figure 6. HOMO-LUMO gaps of small Na clusters estimated by LDA and GW approximations as functions of number of atoms, $N$.
We have performed a numerical calculation based on the all-electron full-potential mixed-basis code to determine LDA wave functions and used them to estimate the HOMO-LUMO gap values of small Na clusters by the GW approximation. The results are shown in figure 6, which almost exactly reproduce the experimentally observed values for all the cases of 2 to 10 atom clusters (differences are not seen, because they are within the size of the circles in the figure) (Ishii et al 2001). In the figure, the GW results obtained by Saito with the jellium mode are also shown. This result by a simple jellium model is already far better than LDA as can be seen in the figure, but quantitative agreement is first obtained by the present numerical calculation. It can easily be seen that LDA could not give quantitative results at all, and the first principle GW approximation gives a very good agreement with experimental values.

\subsection{Chemical reactions traced by solving time- dependent Schrödinger equation directly}

Present day standard of Car-Parrinello method is not a real dynamical process simulation. It only seeks for the most stable structure of materials by changing the matrix diagonalization in estimating the quantum electronic states to time course on the Born-Oppenheimer surface. Since by the first-principles calculation, nothing is known for the first moment, it is necessary to estimate the electronic states and the potential iteratively until a good convergence is achieved. The Car-Parrinello method has, however, been successfully applied to the first principle molecular dynamics simulation, because even on the BornOppenheimer surface we can see eigenmode of vibration of the system and many other interesting physical aspects in materials.

In nuclear physics, time-dependent Schrödinger equation has often been solved as it is, simply because of the fact that the interaction between nucleons is not determined by the first principles. Accordingly, a simple potential is assumed, and it is not a heavy calculation. Recently time-dependent LDA (TDLDA) has been solved by Yabana and Bertsch (1996) to analyse the process of atom collision. It was successful to simulate the scattering cross section to understand excitation mechanism of molecular electronic states through the collision process.

Our aim is to develop the formulation with $a b$ initio determination of electronic states and potentials self consistently within LDA and to apply it to realistic chemical reaction processes (Wu et al, to be published). In general, chemical reaction is a complex process, which has a number of possible branches with various types of rearrangement of atoms. It is a process normally at room temperature or heated at most up to $100^{\circ} \mathrm{C}$. Since the temperature effect is not negligible even in this small range (in the sense of physics), excited states play important role in the reaction. 


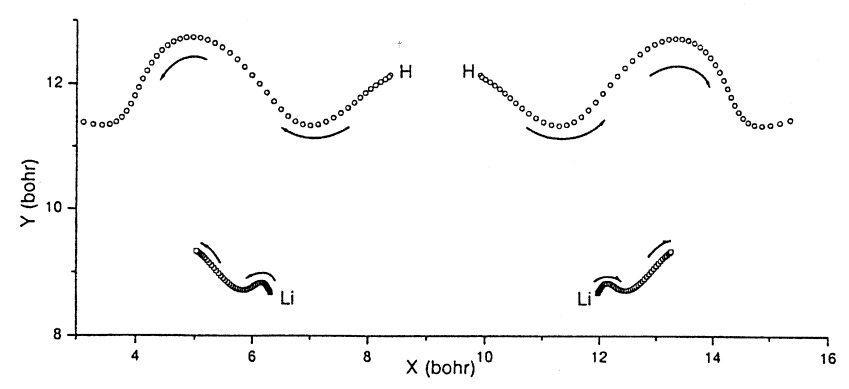

Figure 7. Simulated atom trajectories in $\mathrm{H}_{2}$ and $\mathrm{Li}_{2}$ molecules at an excited state showing the rearrangement reaction between them to form $2 \mathrm{LiH}$.

We have developed a formalism to trace the chemical reaction by integrating effectively the time-dependent Kohn-Sham equation with our all-electron full-potential mixed-basis approach. In this formulation, we start simulation with an initial configuration which is close to a transition state, and integrate the equation to realize both of the time courses to reactants and products.

As an example, the reaction processes of $\mathrm{Li}_{2}$ and $\mathrm{H}_{2}$ in the ground and excited states are simulated by the present formulation. In the ground state, the two molecules are not reactive and start to be separated keeping the configuration of initial two molecules as is shown in figure 7. On the contrary, in an excited state, where two electrons are transferred from HOMO to LUMO levels, $\mathrm{Li}_{2}$ and $\mathrm{H}_{2}$ are gradually separated and to be rearranged to form $2 \mathrm{LiH}$ molecules. The time course of this reaction process is shown in figure 7 , where one can see that $\mathrm{Li}_{2}$ and $\mathrm{H}_{2}$ molecules start to dissociate and $\mathrm{H}$ and $\mathrm{Li}$ atoms are recombinant to form $2 \mathrm{LiH}$. To check the possibility of this process, we have analysed the total energy throughout the process.

\section{Applications}

In this paper, we have introduced conceptually new formulations beyond the present day standards to overcome the difficulties of predictions of new materials which are expected to be used in the highly information oriented society. GW approximation can provide a good estimation for HOMO-LUMO gap in clusters and therefore, it can give us a useful measure to predict optical materials having necessary photo emission wavelength. Time dependent Schrödinger equation solver gives us a power to simu- late chemical reactions beyond the Born-Oppenheimer surface and it is now possible to trace the exchange procedure of atoms in the reaction.

All-electron full-potential formulation is essential to estimate absolute values of the physical and chemical properties. This is the real $a b$ initio calculation scheme in the theoretical condensed matter physics, which can predict new materials properties prior to experimental work. Accordingly this formulation is very useful to be applied in materials research from now on to design new useful materials having desired properties.

\section{Acknowledgements}

The author is thankful to all of his research group members and collaborators who have contributed to this work. Especially, Profs K Parlinski, V Kumar, H Chen, B-L Gu, R-B Tao, X Sun, D-S Wang, L Wille, C-Q Wu, $\mathrm{K}$ Ohno, M Sluiter, J-Z Yu, and $\mathrm{K}$ Esfarjani, Drs $\mathrm{H}$ Mizuseki, Q Sun, T Briere, T Nishimatsu, Y Maruyama, K Shida, M Ishihara, C Majumder, J Wu, J-T Wang, Z-Q Li, A Farajian, R Belosldov, Y Guo, L Zhou, Z Zeng, and Y Hashi, Mrs S Ishii, V Sornthep, and T Morisato, are the researchers who have provided the new concepts and complex and tedious numerical simulations presented in this paper. He is also grateful to the crew of the Information Science Group at the Institute for Materials Research, Tohoku University, for their continuous support of the HITACHI SR8000/H64 supercomputing system.

\section{References}

Hedin L 1965 Phys. Rev. 139 A796

Ishii S, Ohno K, Kawazoe Y and Lowie S 2001 Phys. Rev. B63 1551041

Louie S G, Ho K M and Cohen M L 1979 Phys. Rev. B19 1774

Maruyama Y, Ohno K and Kawazoe Y 1995 Phys. Rev. $B 52$ 2070

Ohno K, Maruyama Y, Esfarjani K, Kawazoe Y, Sato N, Hatakeyama R, Hirata T and Niwano M 1996 Phys. Rev. Lett. 76 3590

Ohno K, Esfarjani K and Kawazoe Y 1999 Computational materials science, Springer Series in Solid State Sciences (Berlin: Springer Verlag) Vol. 129

Sluiter M, Ohno K, Maruyama Y and Kawazoe Y, to be published

Wu J, Ohno K and Kawazoe Y, to be published

Yabana K and Bertsch G F 1996 Phys. Rev. B54 4484 